\title{
Medial Canthoplasty for Epiphora in Dogs: A Retrospective Study of 23 Cases
}

The medical records of 23 dogs that underwent medial canthoplasty for treatment of epiphora were reviewed. The most prevalent breed encountered was the shih tzu. Other affected breeds included the Pekingese, Maltese, toy poodle, and pug. All dogs had epiphora associated with medial canthal trichiasis and/or entropion. Other ocular abnormalities included conjunctivitis, keratitis, pigmentary keratitis, corneal ulceration, globe prolapse, and nasal fold trichiasis. After medial canthoplasty, the epiphora resolved in all dogs.

\section{J Am Anim Hosp Assoc 2006;42:435-439.}

Na Young Yi, DVM

Shin Ae Park, DVM

Man Bok Jeong, MS

Min Su Kim, DVM

Ji Hey Lim, DVM

Tchi Chou Nam, PhD

Kangmoon Seo, PhD

RS

From the Department of

Veterinary Ophthalmology, College of Veterinary Medicine,

Seoul National University,

San 56-1, Sillim 9-dong, Gwanak-gu,

151-742 Seoul, Korea.

Address all correspondence to Dr. Seo.

\section{Introduction}

Epiphora is a common problem in small-breed dogs. ${ }^{1-3}$ The hairs and skin around the medial canthus may become stained and brown because of constant tearing. While tear staining is generally a cosmetic defect, a focal dermatitis may sometimes occur. ${ }^{3}$ Epiphora develops secondary to an impediment of the drainage of tears through the nasolacrimal system or to an overproduction of tears. ${ }^{4}$ Certain anatomical features, such as medial canthal entropion, excess hair growth at the medial canthus (trichiasis), a tight medial palpebral ligament, misplacement of the lower punctum, and close apposition of the eyelids to the globe may also predispose the dog to epiphora. ${ }^{1,3}$ Problems associated with cilia, such as trichiasis and distichiasis, can irritate the conjunctiva and cornea and lead to excessive lacrimation. ${ }^{5}$

Medial canthal trichiasis is common in some breeds, such as the Tibetan spaniel, shih tzu, and Lhasa apso. ${ }^{6}$ The hairs growing from the caruncle may cause epiphora, conjunctivitis, and pigmentary keratitis and may be complicated by macropalpebral fissure, medial entropion, distichiasis, and keratoconjunctivitis sicca. ${ }^{6,7}$ Cryotherapy has been advocated for the treatment of medial canthal trichiasis, where the conjunctiva containing the hairs is frozen with liquid nitrogen using double freezethaw cycles. ${ }^{8}$

Medial lower eyelid entropion causes ventral displacement and functional closure of the lower lacrimal punctum and canaliculus, with subsequent impairment of tear drainage. ${ }^{1}$ To treat medial canthal entropion, a modified Hotz-Celsus resection may be performed. ${ }^{5}$ In this procedure, a triangular piece of skin is excised with the apex of the triangle positioned opposite the lower lacrimal punctum. The wound is then closed with nonabsorbable suture. A tight medial palpebral ligament displaces the medial canthus ventrally, which allows for the overflow of tears onto the face. ${ }^{1}$ Medial canthoplasty has been used to correct canthal entropion and trichiasis, as well as tight medial palpebral ligaments. ${ }^{1}$ Medial canthoplasty can also be used to reduce the palpebral fissure size in brachycephalic dogs. ${ }^{9}$ 
The purpose of this study was to evaluate the surgical outcomes and complications of medial canthoplasty in dogs with epiphora.

\section{Materials and Methods}

Medical records of dogs that underwent a medial canthoplasty for treatment of epiphora from October 2002 to May 2005 were reviewed. All dogs included in the study had no prior surgical treatments for epiphora. Any dogs that received prior electroepilation for distichiasis or nasal fold resection for nasal fold trichiasis were excluded from the study. Complete ophthalmic examinations, including a Schirmer tear test (STT), ${ }^{\text {a }}$ slit-lamp biomicroscopy, ${ }^{\mathrm{b}}$ applanation tonometry, ${ }^{\mathrm{c}}$ fluorescein dye test, ${ }^{\mathrm{d}}$ and an indirect ophthalmoscopy, ${ }^{\mathrm{e}}$ were performed. Epiphora was diagnosed if a dog had an overflow of tears onto the skin near the medial canthus, with a normal STT value. The breed, age, sex, causes of epiphora, and concurrent ocular diseases were investigated. The surgical outcomes and complications were also reviewed. Follow-up clinical evaluations were done postoperatively in all dogs, and owners were also interviewed by telephone.

\section{Surgical Technique}

In all cases, anesthesia was induced with propofol ${ }^{f}$ and maintained with isofluraneg in oxygen. Each dog was positioned in dorsal recumbency, and the head was stabilized with a vacuum pack. $^{\mathrm{h}}$ The surgical procedure was performed with an operating microscope ${ }^{\mathrm{i}}$ using 10-power magnification. The superior and inferior canaliculi were cannulated with 24-gauge polyethylene catheters, ${ }^{j}$ which allowed preservation of both lacrimal puncta. A no. 15 scalpel blade ${ }^{\mathrm{k}}$ was used to make an incision that ran from the center of the medial canthus to the upper lacrimal punctum along the eyelid margin, superficial to the meibomian gland openings [Figure 1A]. A second incision was made on the lower eyelid in the same fashion. The superficial part of the medial palpebral ligament was cut with tenotomy scissors, ${ }^{1}$ and the circumscribed skin was undermined to free it from its orbital attachment. An arrow-shaped wedge of skin near the medial canthus was removed. The hair-bearing mucosa of the caruncle was also removed, and care was taken to preserve the lacrimal puncta and canaliculi [Figure 1B]. The resulting skin wound was closed with 6-0 polyglactin $910,{ }^{\mathrm{m}}$ employing a figure-of-eight pattern [Figure 1C]. One or two simple interrupted sutures were also applied medial to the figure-of-eight suture to enhance skin closure [Figure 1D]. Postoperatively, all dogs received dexamethasone/neomycin/polymyxin B drops ${ }^{n}$ in the operated eyes $q 8$ hours for 14 days; carprofen ${ }^{\circ} 2.2 \mathrm{mg} / \mathrm{kg}$ orally $q 12$ hours for 7 days; and cephalexinp $30 \mathrm{mg} / \mathrm{kg}$ orally $q$ 12 hours for 7 days. The sutures were removed 10 to 14 days after surgery.

\section{Results}

Medial canthoplasty was performed on both eyes of 23 dogs with epiphora. The dogs' ages ranged from 1 to 10 years (mean 5.1 years). The genders of the animals included four males, nine castrated males, nine females, and one spayed female. The most prevalent breed was the shih tzu $(n=15)$. Other affected breeds included the Pekingese $(n=4)$, Maltese $(n=2)$, toy poodle $(n=1)$, and pug $(n=1)$.

A review of the medical records revealed that all dogs developed epiphora at a young age. The causes of epiphora were primarily medial canthal trichiasis and/or entropion. Other ocular abnormalities were found, such as conjunctivitis or conjunctival hyperemia $(n=16)$, keratitis $(n=12)$, pigmentary keratitis $(n=9)$, corneal ulceration $(n=4)$, globe prolapse $(n=4)$, and nasal fold trichiasis $(n=1)$ [Table].

\begin{tabular}{|c|c|}
\hline $\begin{array}{r}\text { Ocular Findings in } 2 \\
\text { Treated by } \mathrm{Mec}\end{array}$ & $\begin{array}{l}\text { ys With Epiphora } \\
\text { anthoplasty }\end{array}$ \\
\hline Reasons for Epiphora & No. of Dogs (\%) \\
\hline Medial canthal trichiasis & $21(91.3)$ \\
\hline Medial canthal entropion & $19(82.6)$ \\
\hline Ocular Abnormalities & No. of Dogs (\%) \\
\hline $\begin{array}{l}\text { Conjunctivitis, } \\
\text { conjunctival hyperemia }\end{array}$ & $16(69.6)$ \\
\hline Keratitis & $12(52.2)$ \\
\hline Pigmentary keratitis & $9(39.1)$ \\
\hline Corneal ulceration & $4(17.4)$ \\
\hline Globe prolapse & $4(17.4)$ \\
\hline Nasal fold trichiasis & $1(4.3)$ \\
\hline Eyelid neoplasm & $1(4.3)$ \\
\hline Anterior uveitis & $1(4.3)$ \\
\hline Glaucoma & $1(4.3)$ \\
\hline Retinal detachment & $1(4.3)$ \\
\hline
\end{tabular}

Examination of the cornea revealed corneal pigmentation (20 eyes), neovascularization (20 eyes), and edema (12 eyes). These corneal changes were found predominantly in the nasal/inferior quadrant near the medial canthus, adjacent to the hairs growing at the caruncle and/or on the eyelids at the medial canthus. Four dogs had a history of globe prolapse associated with macropalpebral fissure. One dog had two eyelid masses near the medial canthus, which were excised and diagnosed as meibomian gland adenomas on 

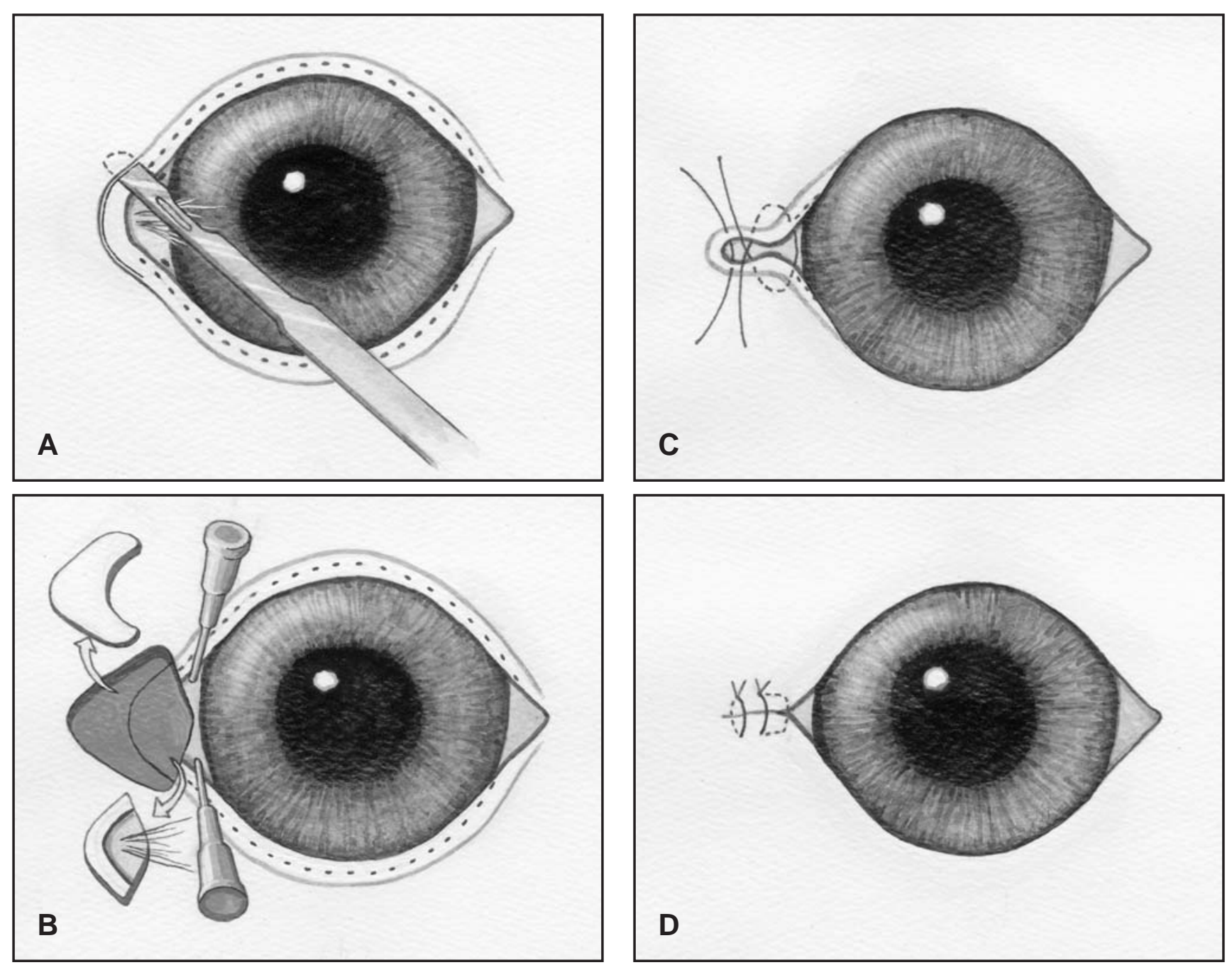

Figures 1A-1D - Schematic diagram showing the medial canthoplasty technique used in the 23 dogs of this study. (A) An incision is made from the center of the medial canthus to the lacrimal punctum along the eyelid margin, superficial to the meibomian gland openings (black dots). (B) An arrow-shaped piece of skin is resected, and the haired mucosa of the caruncle is also removed. The lacrimal puncta have been cannulated in this image. (C) The wound is closed with a figureof-eight suture and additional simple interrupted sutures in the skin. (D) The completed medial canthoplasty, with shortening of the palpebral fissure.

histopathology. Anterior uveitis was noted in one dog, and this dog later developed glaucoma. Retinal detachment was found in one shih tzu.

After medial canthoplasty, all dogs developed moderate to severe chemosis that resolved by the time of suture removal. At the time of suture removal (10 to 14 days), the tearing onto the skin near the medial canthus and tear staining had resolved in all dogs. Recheck examinations were performed for up to 16 weeks (mean 8 weeks) after surgery in all dogs. All corneal ulcers and conjunctivitis resolved within 2 weeks after surgery. Most corneal neovascularization and edema regressed within 1 month after surgery; however, corneal pigmentation showed little improvement during the 16-week observation period. As a result of the medial canthoplasty, the palpebral fissure was shortened approximately 3 to $4 \mathrm{~mm}$ in all dogs. By closing the medial canthus, nasal fold trichiasis resolved in one dog without resection of the nasal fold. Although the operated eyes appeared to be smaller and rounder than before surgery [Figure 2A], 22 (96\%) of the 23 clients whose dogs were rechecked within 3 months were satisfied with the results of medial canthoplasty. Seventeen of the 18 clients who were contacted by telephone ( 5 to 24 months after surgery; mean 12 months) were satisfied with the cosmetic results of the operation. During the follow-up period, epiphora did not recur in any dog.

\section{Discussion}

Breed predisposition for the dogs with medial canthal trichiasis in this study was similar to a previous report. ${ }^{6}$ Medial canthal trichiasis occurred predominantly in brachycephalic breeds.

In 1973, medial conjunctivoplasty was first reported as a treatment for epiphora in $11 \mathrm{dogs} .{ }^{10}$ The procedure involved 

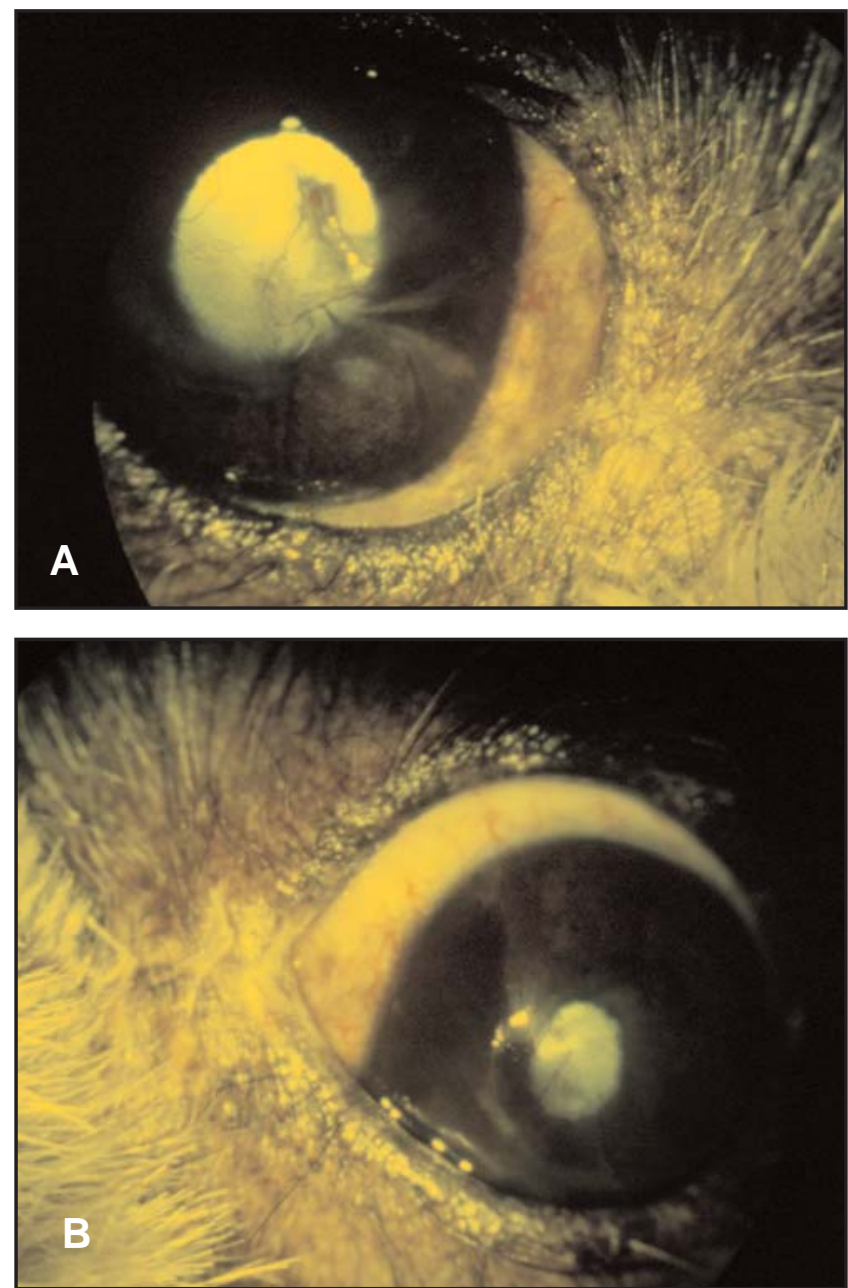

Figures 2A, 2B-Photographs taken 14 days after a medial canthoplasty was performed in a 5-year-old, male shih tzu. (A) Right eye; the medial canthal area looks rounder, and the palpebral fissure is shorter. (B) Left eye; the healed incision at the medial canthus is hairless, and there is no overflow of tears onto the skin.

the removal of aberrant dermis, which reduced the epiphora; however, only six of 11 dogs were permanently cured. In 1979, Jensen described a medial canthoplasty technique that narrowed the palpebral fissure. ${ }^{11}$ The medial canthoplasty technique described in the current study preserves the lacrimal puncta better than the Jensen technique, which sacrifices the upper punctum. The medial canthoplasty described here also appears to be a simpler method than the Jensen technique.

The canthoplasty technique reported here simultaneously removes the excess hair in the caruncle and corrects any medial canthal entropion. The lower eyelid is also loosened by partial resection of the medial palpebral ligament, which may impair tear drainage through the lacrimal punctum. A one-layer skin closure was performed in this study in order to create an empty area by leaving the conjunctival wound open. It was hoped that this open area would provide a larger space for tears to collect and reduce the overflow of tears. Polyglactin 910 was used for the skin closure, because it is soft and less likely to cause corneal irritation.

Another advantage of medial canthoplasty is the shortening of the palpebral fissure, which protects the globe and provides better cosmesis by making any exotropia less noticeable. ${ }^{8}$ Despite the smaller appearance of the eye after surgery, most owners were satisfied with the results. In this study, medial canthoplasty alone prevented nasal fold trichiasis in one dog by eliminating the contact between the cornea and nasal folds by shortening the palpebral fissure. In the event that both macropalpebral fissure and nasal fold trichiasis are present in a dog, medial canthoplasty may be considered if the owner is reluctant to have a nasal fold resection performed.

\section{Conclusion}

Medial canthoplasty was performed in 23 dogs with epiphora. Epiphora resolved in all dogs, and many ancillary corneal changes also improved. The medial canthoplasty technique described here is an effective procedure for the correction of epiphora in toy and brachycephalic dogs.

a Schirmer Tear Test; Schering-Plough Animal Health, Kenilworth, NJ 07033

b SHIN-NIPPON No. 1320884; SHIN-NIPPON Co., Inc., Tokyo, Japan

c Tono-Pen XL; Medtronic, Minneapolis, MN 55432

d Fluorets; Chauvin Pharmaceuticals Ltd, London, England

e Vantage Indirect Ophthalmoscope; Keeler, Berkshire, United Kingdom

f Anepol; Hana Pharm Co., Ltd, Seoul, Korea

g Isoflurane; Rhodia, Bristol, United Kingdom

$\mathrm{h}$ Vacu support (BUSTER); AlfaMedic Ltd, Hong Kong, China

1 Leica M 651; Leica Microsystems GmbH, Wetzlar, Germany

j BD IV catheter; Becton Dickinson \& Co., Helsingborg, Sweden

k Ailee surgical blade; Ailee, Busan, Korea

1 Stevens tenotomy scissors; Bausch \& Lomb, San Dimas, CA 91773

$\mathrm{m}$ Vicryl; Johnson \& Johnson, St-Stevens-Woluwe, Belgium

$n$ Maxitrol; S.A. Alcon-couvreur N.V., Puurs, Belgium

o Rimadyl; Pfizer Animal Health, New York, NY 10017

p Cephalexin; Dongkoo Pharm Co., Ltd, Seoul, Korea

\section{Acknowledgments}

The authors thank the veterinarians who referred the dogs, and illustrator Un Gyu Lim for his drawing in the preparation of the illustrations.

\section{References}

1. Crispin S. The lacrimal system. In: Petersen-Jones S, Crispin S, eds. Brit Small Anim Vet Assoc Manual of Small Animal Ophthalmology. 2nd ed. Quedgeley: Brit Small Anim Vet Assoc, 2002:115-116.

2. Olivero DK. Epiphora. In: Riis RC, ed. Small Animal Ophthalmology Secrets. Philadelphia: Hanley \& Belfus, 2002: 147-152.

3. Slatter D. Fundamentals of Veterinary Ophthalmology. 3rd ed. Philadelphia: WB Saunders, 2001:245-249.

4. Grahn BH. Diseases and surgery of the canine nasolacrimal system. In: Gelatt KN, ed. Veterinary Ophthalmology. 2nd ed. Baltimore: Lippincott Williams \& Wilkins, 1999:570-573. 
5. Bedford PG. Diseases and surgery of the canine eyelids. In: Gelatt $\mathrm{KN}$, ed. Veterinary Ophthalmology. 2nd ed. Baltimore: Lippincott Williams \& Wilkins, 1999:542-558.

6. Petersen-Jones $\mathrm{S}$. The eyelids and nictitating membrane. In: Petersen-Jones S, Crispin S, eds. Brit Small Anim Vet Assoc Manual of Small Animal Ophthalmology. 2nd ed. Quedgeley: Brit Small Anim Vet Assoc, 2002:90-91.

7. Maggs DJ. Conjunctiva. In: Slatter D, ed. Textbook of Small Animal Surgery. 3rd ed. Philadelphia: Elsevier Science, 2003:1346-1347.

8. Munger RJ. Blepharoplastic procedures. In: Riis RC, ed. Small Animal Ophthalmology Secrets. Philadelphia: Hanley \& Belfus, 2002:163-165.
9. Stades FC, Wyman M, Boevé MH, et al. Ophthalmology for the Veterinary Practitioner. Honover: Schlütersche $\mathrm{GmbH} \& \mathrm{Co}$, 1998:74-75.

10. Carter JD. Medial conjunctivoplasty for aberrant dermis of the Lhasa apso. J Am Anim Hosp Assoc 1973;9:242-244.

11. Jensen HE. Canthal closure. Compend Contin Educ Pract Vet 1979;1:735-741. 